\title{
The anti-inflammation effects of resveratrol for patients after oral implantology.
}

\author{
HaSi BaGen, XiaoFei Liu, Jing Han* \\ Department of Oral Implantology, Tianjin Stomatological Hospital, Tianjin, PR China
}

\begin{abstract}
Inflammation is the most common symptom for patients after oral implantology. Evidences have indicated that resveratrol plays ameliorative effects on higher inflammatory factor concentration and metabolic syndrome. In this study, we investigated the therapeutic effects of resveratrol on patients after oral implantology. A total of $\mathbf{1 6 2}$ patients after oral implantology were recruited in this study and inflammatory cytokines were analyzed after treatment with resveratrol or placebo during 4-week observation. RT-qPCR and ELISA were used to the measure changes of inflammatory cytokines in oral implantology patients after resveratrol. Results showed that mRNA and serum levels of IL-1 $\beta$, IL-17A and tumor necrosis factor alpha $(\mathrm{TNF}-\alpha)$ were decreased by Resveratrol treatment in patient after oral implantology. It demonstrated that mRNA and serum levels of IL-2, IL-6 and IL-10 were up-regulated by resveratrol in patient after oral implantology. Notably, we found that resveratrol treatment markedly decreased intercellular adhesion molecule-1 (ICAM-1) and immunoglobulin A1 (IgA1) expression levels for patients after oral implantology.
\end{abstract}

Keywords: Oral implantology, Resveratrol, Inflammation.

\section{Introduction}

Currently, oral implantology has been widely applied for restoration of the missing teeth [1]. A review has introduced the common infectious, the etiology, clinical features, histopathology, and treatment of each oral pathological condition [2]. A study has indicated that the clinical importance of inflammation in bone augmentation procedures in oral implantology [3]. And clinical investigation has reported the effect of piezoelectric surgery implant osteotomy on radiological and molecular parameters of peri-implant crestal bone loss [4]. Although anti-inflammation for patients after oral implantology has been elaborated in previous study, the systematic changes of inflammatory factors the pathological processes in oral implantology have been explored clearly [5].

Resveratrol is multifunctional biological polyphenol that plays ameliorative effects on higher inflammatory factor concentration [6,7]. A study has reported that resveratrol attenuated inflammation and stricture formation in experimental caustic esophageal burns [8]. In addition, previous reports have demonstrated that resveratrol prevented the development of abdominal aortic aneurysm through attenuation of inflammation, oxidative stress, and neovascularization [9]. Furthermore, resveratrol also inhibited inflammation induced by heat-killed Listeria monocytogenes [10]. However, the anti-inflammation effects of resveratrol have not investigated in the progression of oral implantology.
In this study, we investigated the effects of resveratrol for oral implantology in patients after oral implantology. The changes of inflammatory and anti-inflammation factors were measured for oral implantology patients after resveratrol treatment. Findings have indicated that resveratrol treatment decreased inflammatory factors and increased anti-inflammation factors for patients after oral implantology in 4-week treatment.

\section{Materials and Methods}

\section{Patients}

A total of 162 patients after oral implantology (aged 42-68 y old) were recruited in this study in Tianjin Stomatological Hospital between May 2015 and June 2016. The number of male (80) and female (82) patients was approximately equal. All patients after oral implantology were received treatment with resveratrol (2 mg/kg/d, male/female: $42 / 40)$ or placebo (2 $\mathrm{mg} / \mathrm{kg} / \mathrm{d}$, male/female: $38 / 42$ ). Patients with oral implantology were excluded in the present study.

\section{Reverse transcription-quantitative polymerase chain reaction (RT-qPCR) assay}

A total of $5 \mathrm{ml}$ peripheral venous blood was obtained from patient after treatment with resveratrol or placebo. Peripheral blood monouclear cells (hPBMCs) were separated by density gradient centrifugation $(\mathrm{CsCl})$. Total RNA was extracted from hPBMCs cells using RNAzol (Sigma-Aldrich). cDNA was 
synthesized ( $1 \mu \mathrm{g}$ for mRNA) using RNA to cdnaEcoDry ${ }^{\mathrm{TM}}$ Premix (\#639543, Clontech Laboratories, Inc., USA) and quantified using Nanodrop (Thermo Scientific USA, ND-0859). qRT-PCR was performed using Power SYBR ${ }^{\circledR}$ Green PCR Master Mix and Power SYBR ${ }^{\circledR}$ Green RT-PCR Reagents Kit (Applied Biosystem, USA) in 7900HT Fast RealTime PCR System (Applied Biosystem, USA). Changes in relative mRNA expression were calculated by the $2^{-\Delta \Delta \mathrm{Cq}}$ method [11]. The results are expressed as the fold change compared with the control.

\section{ELISA}

Plasma samples were immediately prepared from peripheral venous blood by centrifugation $\left(2,000 \times \mathrm{g}\right.$ at $4^{\circ} \mathrm{C}$ for $\left.10 \mathrm{~min}\right)$. Serum levels of TNF- $\alpha$, IL-6, IL-17A, IL-1 $\beta$, IL-10, IL-2, IgA1 and ICAM-1 were analyzed using ELISA kits according to the manufacturer's protocols.

\section{Statistical analysis}

All data were presented as the mean and standard deviation of triplicate experiments. Unpaired data was determined by Student's t-test. $\quad \mathrm{P}<0.05$ was considered to indicate a statistically significant difference.

\section{Results}

\section{Inflammatory cytokines are decreased by resveratrol for oral implantology patients}

Changes of inflammatory cytokines in the serum and hPBMCs were analyzed in oral implantology patients after 4-week treatment with resveratrol. As shown in Figure 1, the serum levels of IL-1 $\beta$, IL-17A, TNF- $\alpha$ were markedly decreased by resveratrol treatment compared to placebo group. We showed that resveratrol treatment decreased mRNA levels of IL-1 $\beta$, IL-17A, TNF- $\alpha$ in hPBMCs for oral implantology patients. These results indicate that resveratrol can down-regulate inflammatory cytokines for oral implantology patients.
A

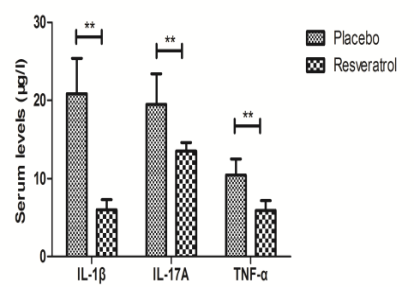

B

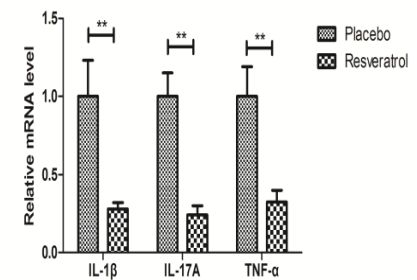

Figure 1. Effects of Resveratrol Inflammatory factors for oral implantology patients. (A) Serum levels of $I L-1 \beta, I L-17 A, T N F-\alpha$ for oral implantology patients after treatment with Resveratrol or placebo; (B) The mRNA levels of $I L-1 \beta, I L-17 A, T N F-\alpha$ in $h P B M C s$ for oral implantology patients after treatment with Resveratrol or placebo.

\section{Resveratrol treatment increases anti-inflammation factors for oral implantology patients}

The changes of anti-inflammation factors were investigated in oral implantology patients after treatment with resveratrol. It demonstrated that serum levels of IL-2, IL-6 and IL-10 were up-regulated by resveratrol in patient after oral implantology (Figure 2A). Results showed that resveratrol treatment also increased mRNA levels of IL-2, IL-6 and IL-10 were increased in hPBMCs in patient after oral implantology compared to placebo (Figure 2B). These results indicate that resveratrol treatment can increase anti-inflammation factors for oral implantology patients.
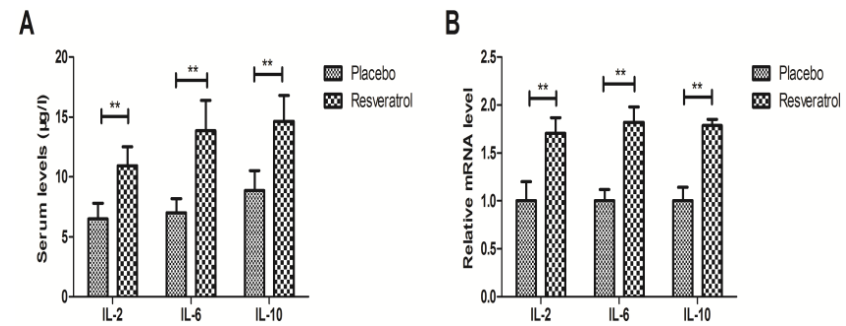

Figure 2. Effects of Resveratrol anti-inflammation factors for oral implantology patients. (A) Serum levels of IL-2, IL-6 and IL-10 for oral implantology patients after treatment with Resveratrol or placebo; (B) The mRNA levels of IL-2, IL-6 and IL-10 in hPBMCs for oral implantology patients after treatment with Resveratrol or placebo.

\section{Resveratrol treatment decreases cytokines expression for patients after oral implantology}

The gene expression levels of cytokines in hPBMCs and serum were assessed in oral implantology patients after treatment with resveratrol. Resveratrol treatment decreased serum levels of ICAM-1 and IgA1 for oral implantology patients (Figure $3 A)$. The mRNA expression levels of ICAM-1 and IgA1 were markedly down-regulated in hPBMCs in oral implantology patients after treatment with resveratrol compared to placebo (Figure 3B). These results suggest resveratrol treatment can decrease cytokines expression for patients after oral implantology.
A

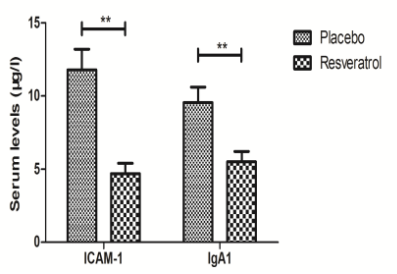

B

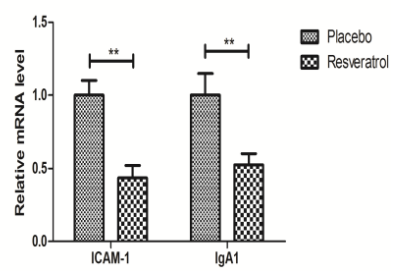

Figure 3. Effects of Resveratrol on cytokines expression for patients after oral implantology. (A) Serum levels of ICAM-1 and IgA1 for oral implantology patients after treatment with Resveratrol or placebo; (B) The mRNA levels of ICAM-1 and IgA1 in hPBMCs for oral implantology patients after treatment with Resveratrol or placebo. 


\section{Discussion}

The clinical assessment of inflammation is necessary to enhance the success rate for patients after oral implantology [12]. Previous studies have found that resveratrol attenuates inflammation in serum and peritoneal macrophages [13-15]. In this study, we analyzed the therapeutic effects of resveratrol for patients after oral implantology. Findings in the current study have indicated resveratrol decreased inflammatory factors and increased anti-inflammation factors in serum and in hPBMCs in oral implantology patients.

Study has showed that resveratrol could inhibit inflammatory factor IL-1 $\beta$-induced stimulation of caspase- 3 and cleavage of PARP in human articular chondrocytes [16]. Results have showed the anti-inflammatory activity of resveratrol and its derivatives and suggested it is a potential clinical relevance in the therapy of inflammatory diseases by decreasing IL-17 production [17]. Resveratrol also decreased TNF- $\alpha$ production and attenuated TNF- $\alpha$-induced activation of coronary arterial endothelial cells [18]. In this study, we observed that resveratrol decreased inflammatory factors IL-1 $\beta$, IL-17A and TNF- $\alpha$ in serum and hPBMCs in oral implantology patients, suggesting resveratrol may be a potential anti-inflammation agent. Our results also found that resveratrol increased antiinflammation factors IL-2, IL-6 and IL-10 in serum in oral implantology patients.

Previous study has reported that serum levels of adhesion molecules ICAM-1 were elevated in patients with vascular inflammation [19,20]. We reported that Resveratrol decreased ICAM-1 serum level, which could down-regulate inflammatory response in cells. A preliminary study has found that Levels of IgA1 were increased in peri-implant fluid and saliva from patients with mucositis [21]. Here, resveratrol treatment markedly decreased IgA1 serum levels and gene expression in hPBMCs in oral implantology patients. Our findings revealed that resveratrol is a potential antiinflammation drug for patients after oral implantology.

In conclusion, the inflammatory factors were decreased and anti-inflammation factors were increased by the treatment of resveratrol for patients after oral implantology. However, future studies should be performed to confirm these results in a larger sample size.

\section{References}

1. Vina JA, El-Alami M, Gambini J, Borras C, Vina J, Penarrocha MA. Application of mesenchymal stem cells in bone regenerative procedures in oral implantology. A literature review. J Clin Exp Dent 2014; 6: 60-65.

2. Meiller TF, Garber K, Scheper M. A review of common oral pathology lesions, with a focus on periodontology and implantology. J Evidence-Based Dent Pract 2012; 12: 254-262.

3. Macedo JP, Pereira J, Vahey BR. Morse taper dental implants and platform switching: The new paradigm in oral implantology. Euro J Dent 2016; 10: 148-154.
4. Peker Tekdal G, Bostanci N, Belibasakis GN, Gurkan A. The effect of piezoelectric surgery implant osteotomy on radiological and molecular parameters of peri-implant crestal bone loss: a randomized, controlled, split-mouth trial. Clin Oral Implants Res 2016; 27: 535-544.

5. Elangovan S, Brogden KA, Dawson DV. Body fat indices and biomarkers of inflammation: a cross-sectional study with implications for obesity and peri-implant oral health. Int J Oral Maxillofacial Implants 2014; 29: 1429-1434.

6. Chen S, Zhao X, Ran L. Resveratrol improves insulin resistance, glucose and lipid metabolism in patients with non-alcoholic fatty liver disease: a randomized controlled trial. Dig Liver 2015; 47: 226-232.

7. Ma T, Tan MS, Yu JT, Tan L. Resveratrol as a therapeutic agent for Alzheimer's disease. BioMed Res Int 2014; 2014: 350516.

8. Uguralp S, Irsi C, Aksoy T, Karabulut AB, Kirimlioglu H, Mizrak B. Resveratrol attenuates inflammation and stricture formation in experimental caustic esophageal burns. Pediatr Surg Int 2008; 24: 425-430.

9. Kaneko H, Anzai T, Morisawa M. Resveratrol prevents the development of abdominal aortic aneurysm through attenuation of inflammation, oxidative stress, and neovascularization. Atherosclerosis 2011; 217: 350-357.

10. Park DW, Kim JS, Chin BR, Baek SH. Resveratrol inhibits inflammation induced by heat-killed Listeria monocytogenes. J Med Food 2012; 15: 788-794.

11. Livak KJ, Schmittgen TD. Analysis of relative gene expression data using real-time quantitative PCR and the 2 (-Delta Delta C (T)) method. Methods 2001; 25: 402-408.

12. Sulzer TH, Bornstein MM, Buser D. Indications for oral implantology in a referral clinic. A three-year retrospective analysis of 737 patients with 1176 implants. Schweizer Monatsschrift fur Zahnmedizin 2004; 114: 444-450.

13. Inoue H, Nakata R. Resveratrol targets in inflammation. Endocrine Metab Immune Disorders Drug Targets 2015; 15: 186-195.

14. Park HG, Lee YR, Jun JK, Lee WL. Exercise training is more effective than resveratrol supplementation on alleviation of inflammation in peritoneal macrophages of high fat diet mice. J Exercise Nutr Biochem 2014; 18: 79-87.

15. Li J, Xie C, Zhuang J. Resveratrol attenuates inflammation in the rat heart subjected to ischemia-reperfusion: Role of the TLR4/NF-kappaB signaling pathway. Mol Med Rep 2015; 11: 1120-1126.

16. Shakibaei M, John T, Seifarth C, Mobasheri A. Resveratrol inhibits IL-1 beta-induced stimulation of caspase-3 and cleavage of PARP in human articular chondrocytes in vitro. Ann New York Acad Sci 2007; 1095: 554-563.

17. Lanzilli G, Cottarelli A, Nicotera G, Guida S, Ravagnan G, Fuggetta MP. Anti-inflammatory effect of resveratrol and polydatin by in vitro IL-17 modulation. Inflammation 2012; 35: 240-248.

18. Csiszar A, Smith K, Labinskyy N, Orosz Z, Rivera A, Ungvari Z. Resveratrol attenuates TNF-alpha-induced 
activation of coronary arterial endothelial cells: role of NFkappaB inhibition. Am J Physiol 2006; 291: 1694-1699.

19. Jublanc C, Beaudeux JL, Aubart F. Serum levels of adhesion molecules ICAM-1 and VCAM-1 and tissue inhibitor of metalloproteinases, TIMP-1, are elevated in patients with autoimmune thyroid disorders: relevance to vascular inflammation. Nutr Metab Cardiovas Dis 2011; 21: 817-822.

20. Carpagnano GE, Spanevello A, Sabato R. Systemic and airway inflammation in sleep apnea and obesity: the role of ICAM-1 and IL-8. J Labor Clin Med 2010; 155: 35-43.

21. Moura CC, Soares PB, Vieira AE, Souza MA, Dechichi P. Levels of immunoglobulin A1 in peri-implant fluid and saliva from patients with mucositis: a preliminary study. Braz Dent J 2013; 24: 380-384.

\section{*Correspondence to}

Jing Han

Department of Oral Implantology

Tianjin Stomatological Hospital

Tianjin

PR China 